\title{
Influence of calcineurin inhibitors and genetic polymorphism of transporters on enterohepatic circulation and exposure of mycophenolic acid in Chinese adult renal allograft recipients
}

\author{
Sha-Sha Sun ${ }^{1}$, Kun Shao ${ }^{1}$, Jia-Qian Lu${ }^{1}$, Hui-Min An ${ }^{1}$, Hao-Qiang Shi ${ }^{1}$, Zhou Peijun ${ }^{1}$, and \\ Bing Chen ${ }^{2}$ \\ ${ }^{1}$ Shanghai Jiao Tong University Medical School Affiliated Ruijin Hospital \\ ${ }^{2}$ Shanghai Jiao Tong University Medical School Affiliated Ruijin Hospital
}

January 9, 2022

\begin{abstract}
Aim: Study the influence of calcineurin inhibitors (CNI) and genetic polymorphisms of transporters on enterohepatic circulation (EHC) of mycophenolic acid (MPA) in Chinese adult renal allograft recipients and estimate the effect of various covariates on prediction performance of MPA AUC0-12h. Method: MPA concentrations of 125 Chinese patients were collected 0-12 hours after administration. Genotypes of transporters were determined in 64 patients. The influence of type of CNI and genetic polymorphisms on MPA exposure was studied. Shapley additive explanations method was used to study the impact of sampling times and covariates related to EHC on AUC0-12h. Extreme gradient boosting (XGboost) machine learning-based model was established to predict AUC0-12h. Results: Dn-AUC6-12h was significantly lower in patients co-administered with CsA $(\mathrm{P}<0.05)$. When co-administered with TAC, for SLCO1B1 T521C or ABCC C-24T, patients with wild-type genotype had significantly higher dn-AUC6-12h ( $\mathrm{P}<0.05)$. Patients with SLCO1B3 334T/699G alleles had significantly lower dn-AUC6-12h than homozygotes $(\mathrm{P}=0.004)$. No significant difference was found in CsA subgroup. For estimating AUC0-12h, C0h, C2h, $\mathrm{C} 8 \mathrm{~h}$, type of CNI, transporters genotypes and the difference between $\mathrm{C} 0 \mathrm{~h}$ and $\mathrm{C} 2 \mathrm{~h}$ were retained in the final model, which had good prediction performance ( $\mathrm{r} 2=0.9739)$. Conclusion: Patients co-administered with CsA had lower MPA EHC than those who received TAC. MPA EHC is affected by ABCC2 C-24T, SLCO1B3 T334G/G699A, and SLCO1B1 T521C genotypes in patients treated with TAC. Type of CNI and genetic polymorphisms of transporters can improve prediction performance of MPA AUC0-12h estimating model, developed using XGboost machine learning method.
\end{abstract}

\section{Hosted file}

MainDocument.docx available at https://authorea.com/users/454573/articles/552155-influenceof-calcineurin-inhibitors-and-genetic-polymorphism-of-transporters-on-enterohepaticcirculation-and-exposure-of-mycophenolic-acid-in-chinese-adult-renal-allograftrecipients

\section{Hosted file}

Tables.docx available at https://authorea.com/users/454573/articles/552155-influenceof-calcineurin-inhibitors-and-genetic-polymorphism-of-transporters-on-enterohepaticcirculation-and-exposure-of-mycophenolic-acid-in-chinese-adult-renal-allograftrecipients 

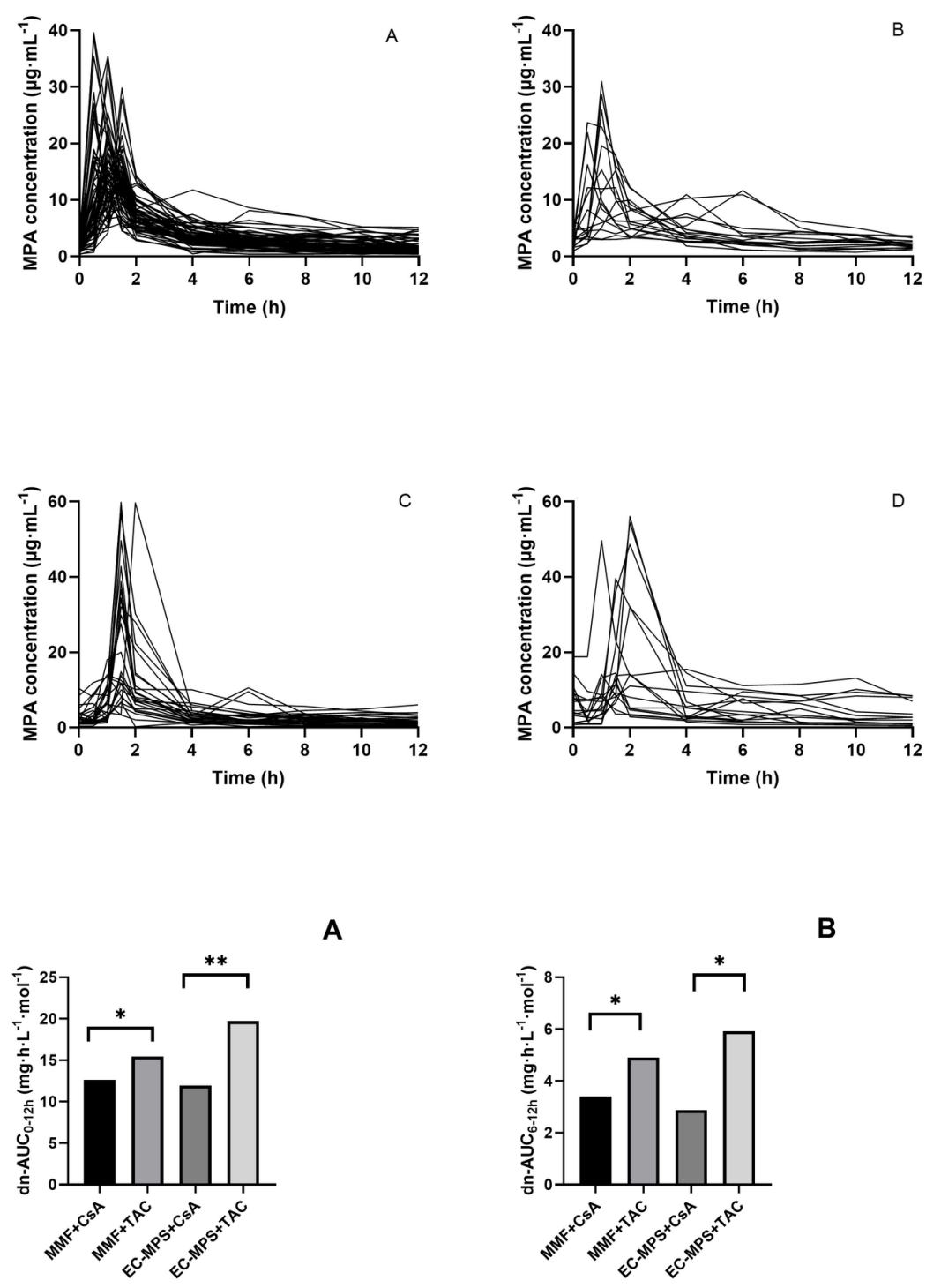

A
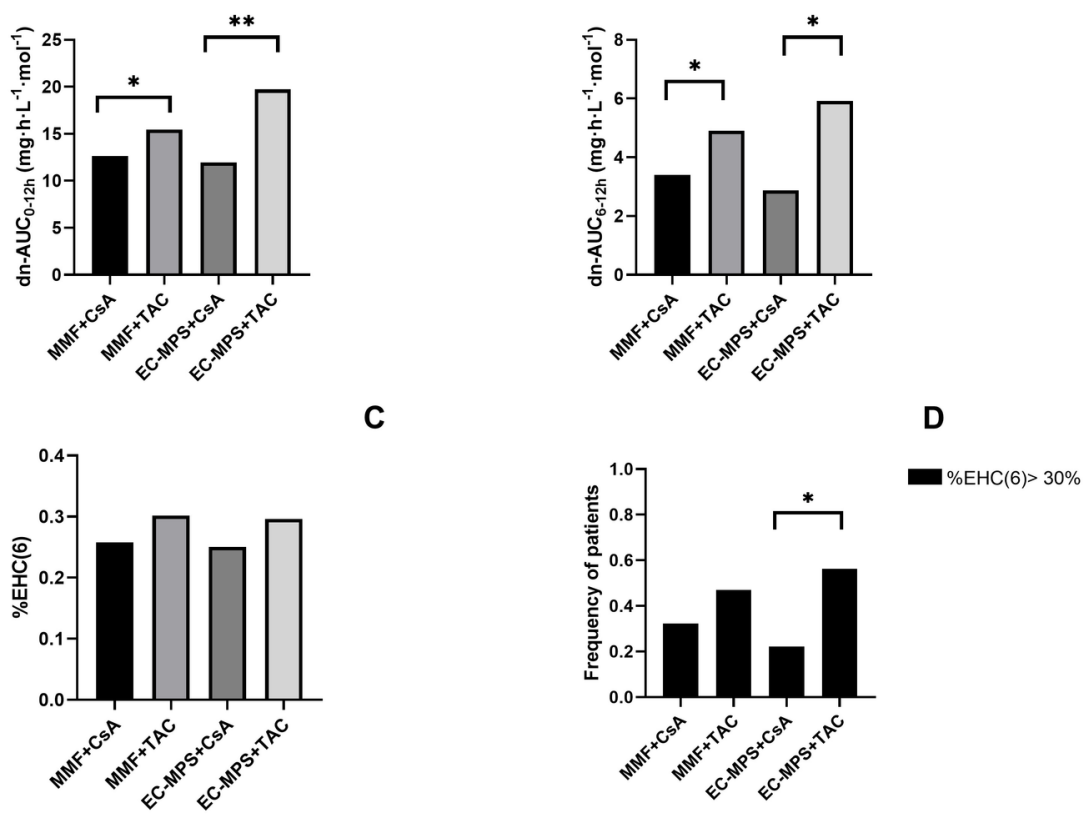

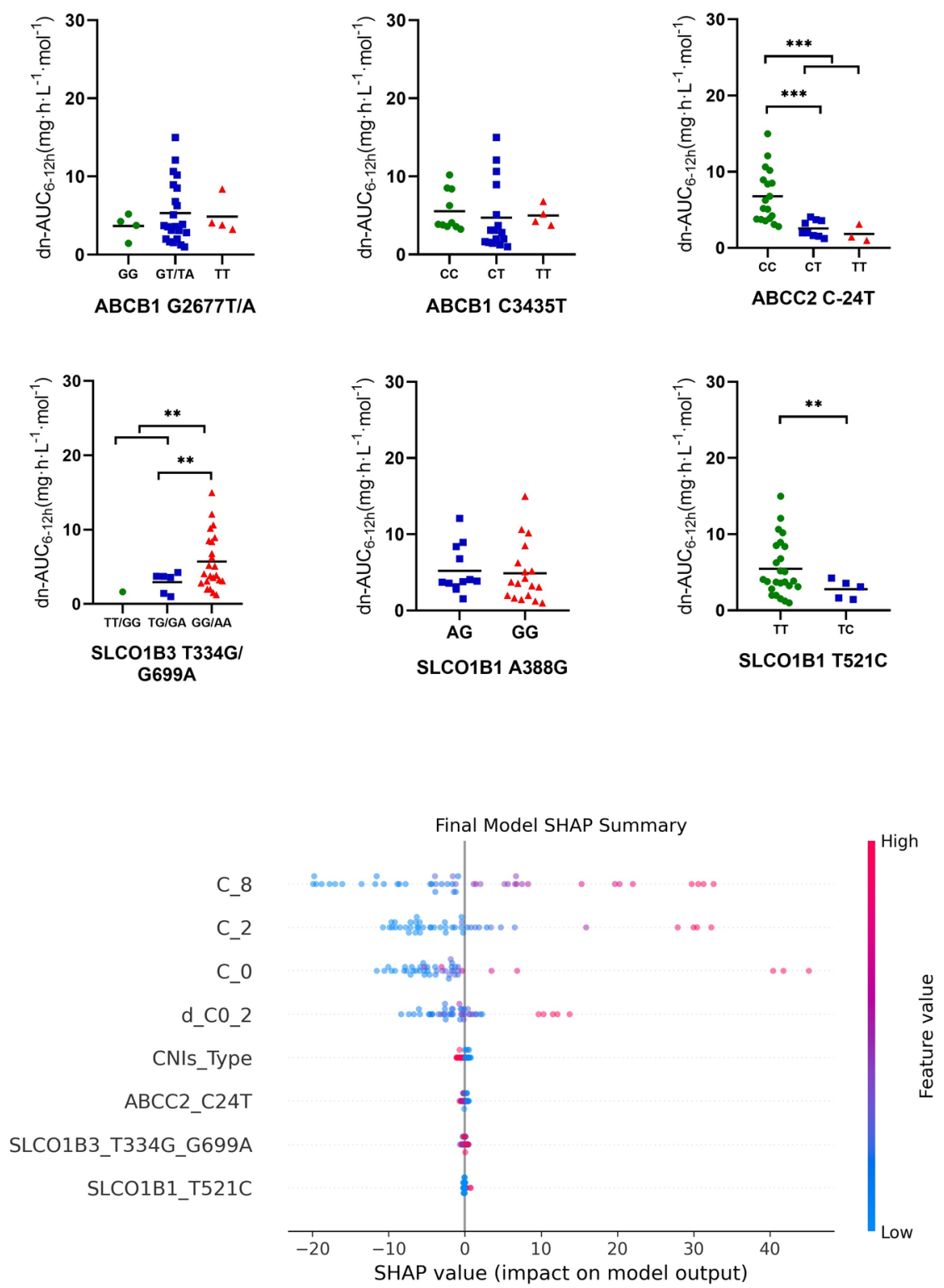

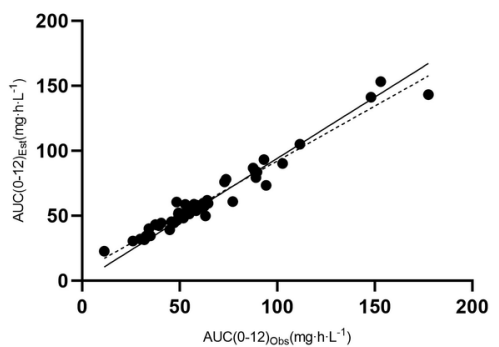

A

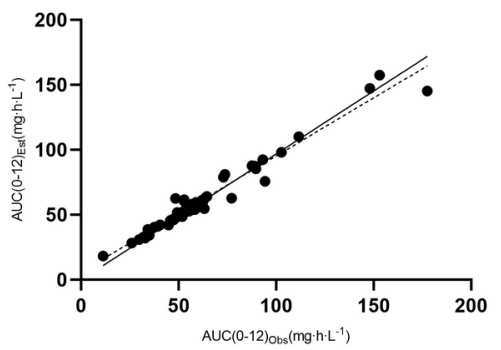

B 

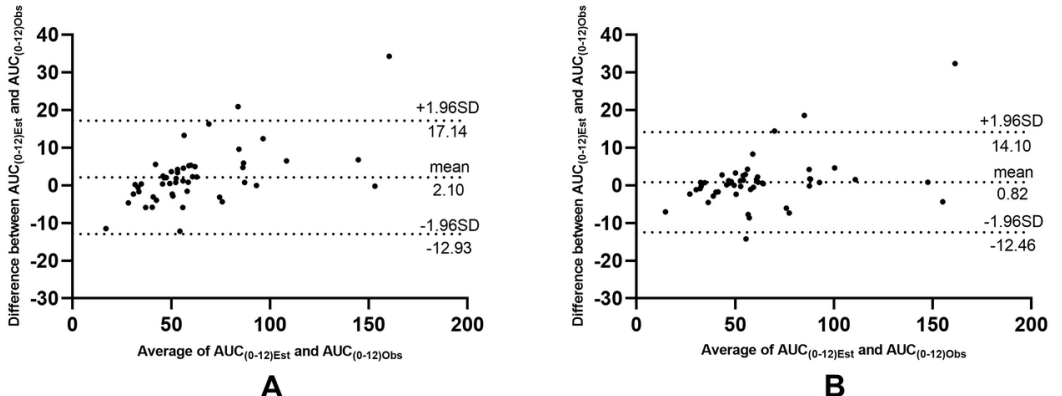